\title{
Optical Light Curves of Supernovae
}

\author{
Bruno Leibundgut ${ }^{1}$ and Nicholas B. Suntzeff ${ }^{2}$ \\ 1 European Southern Observatory, Karl-Schwarzschild-Strasse 2, D-85748 Garching, \\ Germany \\ 2 Cerro Tololo Inter-American Observatory, Casilla 603, La Serena, Chile
}

\begin{abstract}
Photometry is the most easily acquired information about supernovae. The light curves constructed from regular imaging provide signatures not only for the energy input, the radiation escape, the local environment and the progenitor stars, but also for the intervening dust. They are the main tool for the use of supernovae as distance indicators through the determination of the luminosity.

The light curve of SN1987A still is the richest and longest observed example for a core-collapse supernova. Despite the peculiar nature of this object, as explosion of a blue supergiant, it displayed all the characteristics of type II supernovae. The light curves of type $\mathrm{Ib} / \mathrm{c}$ supernovae are more homogeneous, but still display the signatures of explosions in massive stars, among them early interaction with their circumstellar material.

Wrinkles in the near-uniform appearance of thermonuclear (type Ia) supernovae have emerged during the past decade. Subtle differences have been observed especially at near infrared (NIR) wavelengths. Interestingly, the light curve shapes appear to correlate with a variety of other characteristics of these supernovae.

The construction of bolometric light curves provides the most direct link to theoretical predictions and can yield sorely needed constraints for the models. First steps in this direction have been already made.
\end{abstract}

\section{Physics of Supernova Light Curves}

The temporal evolution of the energy release by supernovae (SNe) is one of the major sources of information about the nature of these events. The brightness information is relatively easy to obtain and, hence, light curves have been one of the main stays of supernova research. It is not only the light curves themselves, but also the absolute luminosity and the color evolution that have provided major insights into the supernova phenomenon. Through light curves it has been possible to distinguish between progenitor models, infer some aspects of the progenitor evolution, measure the power sources, detail the explosion models, and probe the local environment of the supernova explosions.

The observational data have been substantially increased over the last decade (for a status before 1990 see [51]). In particular, there are now large databases with light curve data for type Ia supernovae (SNIa). The data on type II supernovae has been extensively expanded as well, but there is still a clear lack of light curves for peculiar objects. Over the last decade the supernova family has

^ to be published in: "Supernovae and Gamma Ray Bursters", Lecture Notes in Physics (http://link.springer.de/series/lnpp) 
further acquired new members and subclassifications (see the chapter by Turatto in this volume).

The energetic display of a supernova can have several different input sources. The most important power comes in almost all cases from the radioactive decay of material newly synthesized in the explosion. The major contributor is ${ }^{56} \mathrm{Ni}$, the main product of burning to nuclear statistical equilibrium at the temperatures and densities encountered in supernovae. This nucleus is unstable and decays with a half-life of 6.1 days due to electron capture to ${ }^{56} \mathrm{Co}$ emitting $\gamma$-photons with energies of $750 \mathrm{keV}, 812 \mathrm{keV}$, and $158 \mathrm{keV}$. The cobalt isotope is also unstable and decays with a half-life of 77.1 days through electron capture $(81 \%)$ and $\beta$-decay $(19 \%)$ to ${ }^{56} \mathrm{Fe}$. In this process $\gamma$-photons with energies of $1.238 \mathrm{MeV}$ and $847 \mathrm{keV}$ are emitted. The kinetic energy of the electrons is about $600 \mathrm{keV}$ (for more details on the radioactive decays see $[3,26,78]$ ). The $\gamma$-rays are downscattered or thermalized in the ejecta until they emerge as optical or NIR photons $[2,48,85]$.

The light curves depend on the size and mass of the progenitor star and the strength of the explosion. Additional energy input, which results in modulations of the emerging radiation, comes from shock cooling, recombination of the ionized ejecta, collision of the shock with circumstellar medium (CSM) and possible accretion onto a compact remnant. Light curves are further shaped by the time-variable escape fraction of $\gamma$-rays, dust formation and absorption in the interstellar medium. In some cases, forward scattered light can change the light curves, e.g., through light echos and fluorescence of nearby gas ionized by the X-ray/UV shock breakout of the explosion.

With this panoply of different energy contributors and modulators, light curve displays are very rich indeed. Despite the plethora of possibilities, light curves of different supernova types are rather distinct, although not sufficiently so for a solid classification. They are, however, important tools to learn about the physics of supernovae.

Light curves are discussed in $[37,51,56,80,81]$. Reviews concentrating more specifically on SNIa light curves can be found in $[57,58,73,107]$, while the light curves of core-collapse supernovae have mostly been summarized in relation to SN1987A [4,72]. Additional well-sampled data sets are available for SN1993J, SN1998S and SN1999em (see references below).

The following sections give a brief overview of observational data sources $(\S 2)$, describe the light curves of the main supernova types (core-collapse supernovae in $\S 3$ and thermonuclear supernovae in $\S 4$ ) and the physics behind the light curve shapes. We will discuss bolometric light curves in $\S 5$ before we summarize in $\S 6$.

\section{Observations}

With modern area detectors, light curves of supernovae have become much easier to assemble. While early light curves have been compiled from photographic plates $[60,82,84]$ observations are now recorded with CCDs. The increased sensitivity has allowed astronomers to successfully move to smaller-size 
telescopes and to improve the temporal sampling. In parallel, the move to more robotic telescopes has increased the number of supernova discoveries tremendously (see, e.g., the chapter by Cappellaro in this volume). There are many observational programs for supernovae currently in progress (for a description of some see [58]), which contribute photometry for many supernovae. Most successful are efforts with semi-automated telescopes. The robotic telescopes of the Lick Observatory and Tenagra Observatory Supernova Search (LOTOSS) have discovered and followed many supernovae in the last few years $[65,90]$. At the Cerro Tololo Inter-American Observatory (CTIO) the Yale-AURA-Lisbon-Ohio (YALO) telescope has regularly provided light curves for nearby supernovae (see, e.g., [54,112]). light curves have been further contributed by the Padova group (see, e.g., $[9,97,116,117]$ and the results of the Harvard-Smithsonian Center for Astrophysics (CfA) team [50,92]). There are additional contributions on SN1993J $[8,11,27,64,74,90,120]$ and more recently SN1998S [32] and SN1999em [45,63]. Many amateur groups are also collecting supernova light curves. These data are mostly maintained on Web sites (see [58] for a small collection of sites).

Infrared observations are still rare. A complete compilation of the available photometry for SNIa before 2000 has been provided by Meikle [73]. More data are being added (see, e.g., [46,54]). For core-collapse supernovae complete light curves are available only for SN1987A [108,110], SN1993J [120], SN1998S [32], and SN1999em [45]. There are several programs starting up that will concentrate on NIR light curves with robotic telescopes.

\section{Core Collapse Supernovae}

Once the shock, which results from the reversal of the core collapse, breaks out at the surface of the progenitor star the fireworks begin. The rapid evolution of the core burning just before the collapse is hidden from the surface due to the long time scales in the atmosphere. The brightness of the shock break out is mostly determined by the temperature in the shock and the size of the progenitor star $[20,31,53]$. This early peak lasts typically from a few hours to a couple of days and has been observed only for SN1987A [4], SN1993J [90], and SN1999em $[45,63]$. After a rapid, initial cooling the supernova enters a phase when its temperature and luminosity remain fairly constant $[29,45,63]$. For supernovae with large progenitors the resulting light curve shows a plateau, while the evolution of supernovae from smaller stars first exhibits a decline before the supernova brightens again to reach the plateau. Examples for the former are SN1990E [98] and SN1999em [45,63], while the type II SN1987A (see, e.g., Fig. 1) together with the $\mathrm{SNIb} / \mathrm{c}$ belong to the latter (see, e.g., [56]). The plateau originates from a balance between the receding photosphere in the expanding ejecta [29]. During this phase the supernova is powered by the recombination of hydrogen previously ionized in the supernova shock. The length of the plateau phase is determined by the depth of the envelope (i.e., the envelope mass and the explosion energy), which is reflected in the expansion velocity of the ejecta $[19,89]$. For 


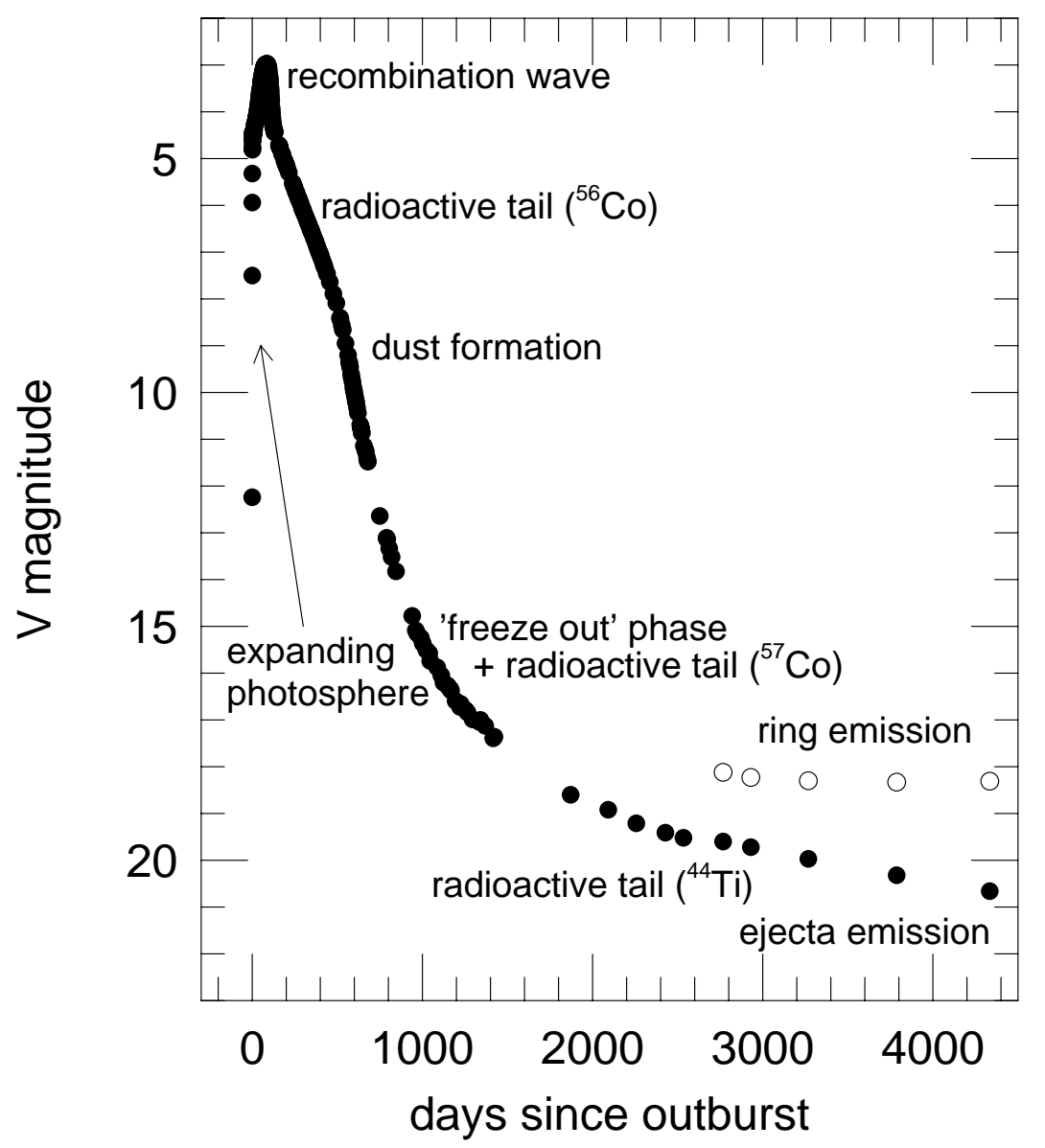

Fig. 1. V light curve of SN1987A. The various phases are labeled.

some objects this plateau phase is conspicuously absent [80]. Most prominent among these are SN1979C [15] and SN1980K [7].

Once the photosphere has receded deep enough, additional heating from the radioactive decay of ${ }^{56} \mathrm{Ni}$ and ${ }^{56} \mathrm{Co}$ extends the plateau for a brief time [123]. Afterwards the light curve is powered solely by the radioactive decay in the remaining nebula. The $\gamma$-rays are captured in the ejecta and converted into optical photons, which can escape freely. At this moment the supernova light curve drops onto the "radioactive tail." This happens typically after about 100 days (see Fig. 1).

For a complete trapping of the $\gamma$-rays the luminosity of the late decline gives an indication of the amount of ${ }^{56} \mathrm{Ni}$ and ${ }^{56} \mathrm{Co}$ decays powering the light curve $[12,99]$. This can be checked with the decline rate of the bolometric light curve, which should reflect the ${ }^{56}$ Co decay time. 
From such measurements a rather large range of nickel masses has been derived $[99,103,116]$. These phases are especially interesting as they may show signatures of significant fallback of the inner explosion material onto the forming compact object, neutron star or black hole, in the explosion $[6,10,122]$.

Especially for SNIb/c the decline rates are steeper $[21,90,104,105]$ at these times, which is an indication that some of the $\gamma$-rays escape from the ejecta without any energy deposition.

Very few objects have been followed beyond about 200 days and the situation has not changed very much since about 10 years ago [80,113]. The photometry of such objects becomes very difficult as they fade into the glare of the underlying galaxy. The remarkable exception is, of course, SN1987A on which all very late phase information is based. This supernova suffered from dust forming within the ejecta, which resulted in an increase of the decline rate in the optical as light was shifted to the infrared [111]. This occurred after about 450 days and could also be observed as a shift of emission lines towards the blue as the redder parts of the lines were absorbed. After about 800 days the light curves started to flatten again [111] due to energy release of ionized matter [40]. This so-called "freeze-out" stems from tenuous material which was ionized during the original explosion but recombines on time scales longer than the expansion time. At later times, the flattening is caused by the energy input from long-lived ${ }^{57} \mathrm{Co}$ (half-life of 270 days) and ${ }^{44} \mathrm{Ti}$ which has a half-life of about 60 years [26].

As is apparent from Fig. 1, the very late times are, in fact, dominated by the emission from the circumstellar inner ring, which was ionized by the shock breakout [39]. Around 1500 days after the explosion the ring emission is stronger than that from the supernova ejecta itself.

The closeness of SN1987A has permitted us to resolve the ring emission and also light echos from interstellar dust from the supernova ejecta [109,124]. For any other supernova these contributions can not be separated and would influence the light curve shape.

Some supernovae do not follow the path described above. They often have a much slower evolution and also display narrow lines in their spectra (see, e.g., $[37,41])$. These objects remain bright for a long time and must be powered by a different energy source. The mostly likely explanation for these objects is interaction of the shock with a dense circumstellar medium (CSM). In this process, kinetic energy is converted to light and hence an additional energy source can be tapped for the light curve. Since many of these objects are dominated by line emission and the line strengths critically depend on density and composition of both the supernova ejecta and the circumstellar medium (CSM), filter light curves can vary significantly from object to object. A few classical cases are known so far: SN1978K [96], SN1986J [61,95], SN1988Z [114] and SN1995N [41].

Of similar nature are probably also objects which can be observed for decades after their explosions although at a much lower luminosity. The prime examples are SN1979C [35,119], SN1980K [34,36,61], SN1970G [33], and SN1957D [68,69]. A summary of the observational characteristics of these objects can be found in Leibundgut [55] and the detailed physics of the SN-circumstellar medium (CSM) 
interaction is given in the chapter by Chevalier and Fransson in this volume. It is noteworthy that all these objects also emit radio waves (see the chapter by Sramek and Weiler in this volume).

On no occasion has it been possible, so far, to observe the emergence of a pulsar within a supernova. Even for SN1987A the data do not require any input from a pulsar. Some objects which have been observed for decades, like SN1957D, SN1970G, SN1979C, SN1980K, still do not require the energy input corresponding to the expectations from a pulsar powered plerion (see, e.g., [55]). It is more likely that these objects are powered by interaction of the shock with the circumstellar medium (CSM). In all of these cases the spectrum clearly shows that we are still observing supernova light and not an underlying stellar association. This is further supported by changes in the light curves as observed for SN1957D [69] and SN1980K [36].

\section{Thermonuclear Supernovae}

The observational situation for type Ia SNe is quite different. There have been several focused searches for SNIa, which have produced large sets of well-sampled light curves (see, e.g., $[42,44,46,50,66,73,88,91,92,97,107,112])$. These data samples have produced a detailed view of SNIa. A recent summary of SNIa light curves can be found in Leibundgut [58] and Meikle [73].

The incineration of a white dwarf, which is the most favored model today (see, e.g., $[47,123]$ ), does not predict an observable shock breaking out at the surface. The rise in brightness is due to the increase in size of the ejecta and lasts for almost three weeks $[1,23,42,93]$ with the color and temperature rather constant. The earliest observed supernovae are SN1990N and SN1998bu, which were observed about 17 days before maximum $[58,93]$. The light curves are shaped by the progressing diffusion of photons out of the ejecta (see, e.g., [85]).

The maximum is reached first at NIR wavelengths [24,73] and is followed a few days later in the optical. While the blue light curves display a monotonic decrease of the brightness for the first month after maximum, the NIR bands $\mathrm{I}, \mathrm{J}, \mathrm{H}$, and $\mathrm{K}$ display a prominent second maximum after about 20 days for most SNIa [30,73], which is often also observed as "shoulders" in the V and R filter light curves. This second maximum is conspicuously missing for objects of the type Ia faint subclass with SN1991bg and SN1999de as the most prominent examples $[38,62,77,115]$.

SNIa show a strong color evolution towards the red through the maximum phase. Despite the difficulty that this poses for an exact measurement, the intrinsic color of SNIa appears to be very uniform [88] and this is often used to determine the amount of reddening towards the supernova.

After about 40 days the light curves settle onto an exponential (in luminosity) decline for several months. This has been interpreted as the optically thin phase when the ejecta nebula captures fewer and fewer $\gamma$-rays and the optical and NIR light curves decline faster than the ${ }^{56}$ Co decay rate (see, e.g., $\left.[49,59,85]\right)$. After about 150 days the light curves change slope once more (see, e.g., [58]) when 
the importance of the positron channel in the ${ }^{56}$ Co decay sets in (see, e.g., $\S 1$, $[5,75]$. The decline at these phases should tell us about the magnetic fields in the explosion as they determine whether the positrons are captured or escape the ejecta $[22,75,94]$. Currently, the best estimate of these late light curves predicts a positron escape similar to that of the photons [76].

Light echos can start to dominate SNIa light curves several hundred days after the explosion. There are now at least two objects with clear signatures of light echos, SN1991T [100,106] and SN1998bu [18]. Their light curves have flattened almost completely as the peak light is scattered off nearby dust clouds and, since we observe time and intensity integrated light, the brightness does not change until the edge of the dust layer is reached or the scattering angle increases to the point where the scattering efficiency decreases. High spatial resolution imaging shows rings around these supernovae ([106] and Garnavich, private communication) very similar to the ones observed around SN1987A.

Despite the highly complicated hydrodynamics and the radiative transport in SNIa ejecta the bolometric light curves can provide important insights. Most of the emission from SNIa is emitted in the optical and NIR region [23,107]. By sampling the emission from the atmospheric cutoff near $3600 \AA$ to $1 \mu \mathrm{m}$ we are capturing about $80 \%$ of the energy emitted by these objects outside the $\gamma$-ray region. Although the color changes significantly through the observable life of a SNIa, not much light is emitted in the near-UV (see, e.g., $[52,57,107]$ ) or the infrared [23]. In fact, the V light curve is a rather good surrogate of the bolometric light curve after maximum [24]. In particular, during the late declines the $\mathrm{V}$ light curves have been used to calculate the Ni mass synthesized in the explosions [17] and also to estimate the positron escape [76].

Fig. 2 illustrates the connection between observed bolometric light curves (dotted line - constructed from observations of SN1992bc) and theoretical models. The expected decay lines for ${ }^{56} \mathrm{Ni}$ and ${ }^{56} \mathrm{Co}$ are indicated by the thin, gray lines. At early times all the energy is trapped in the ejecta and as the surface increases the brightness increases as well. Around maximum the energy released is almost identical to the decay energy from ${ }^{56} \mathrm{Ni}$ and ${ }^{56} \mathrm{Co}$ combined. This has been pointed out long ago by Arnett [2] and has been confirmed by Pinto and Eastman [85]. A compilation of typical values of early models has been given in Branch [14]. This is an important feature of SNIa and can be used to measure the total amount of radioactive material synthesized in the explosion (see, e.g., [24]). For a brief time after maximum the energy output from the supernova exceeds the prediction from a complete trapping of the radioactive energy while "old" photons still leak out at the surface. At later phases more and more $\gamma$ rays are lost and the decline is faster than the line indicating a full trapping (dash-dotted). The dashed line indicates a light curve in which no energy from the original $\gamma$ decays is converted to optical light. Only when $3 \%$ of the $\beta$ decay sets in can there be some levelling of the light curve. The observed light curve evolves between these extreme cases. 


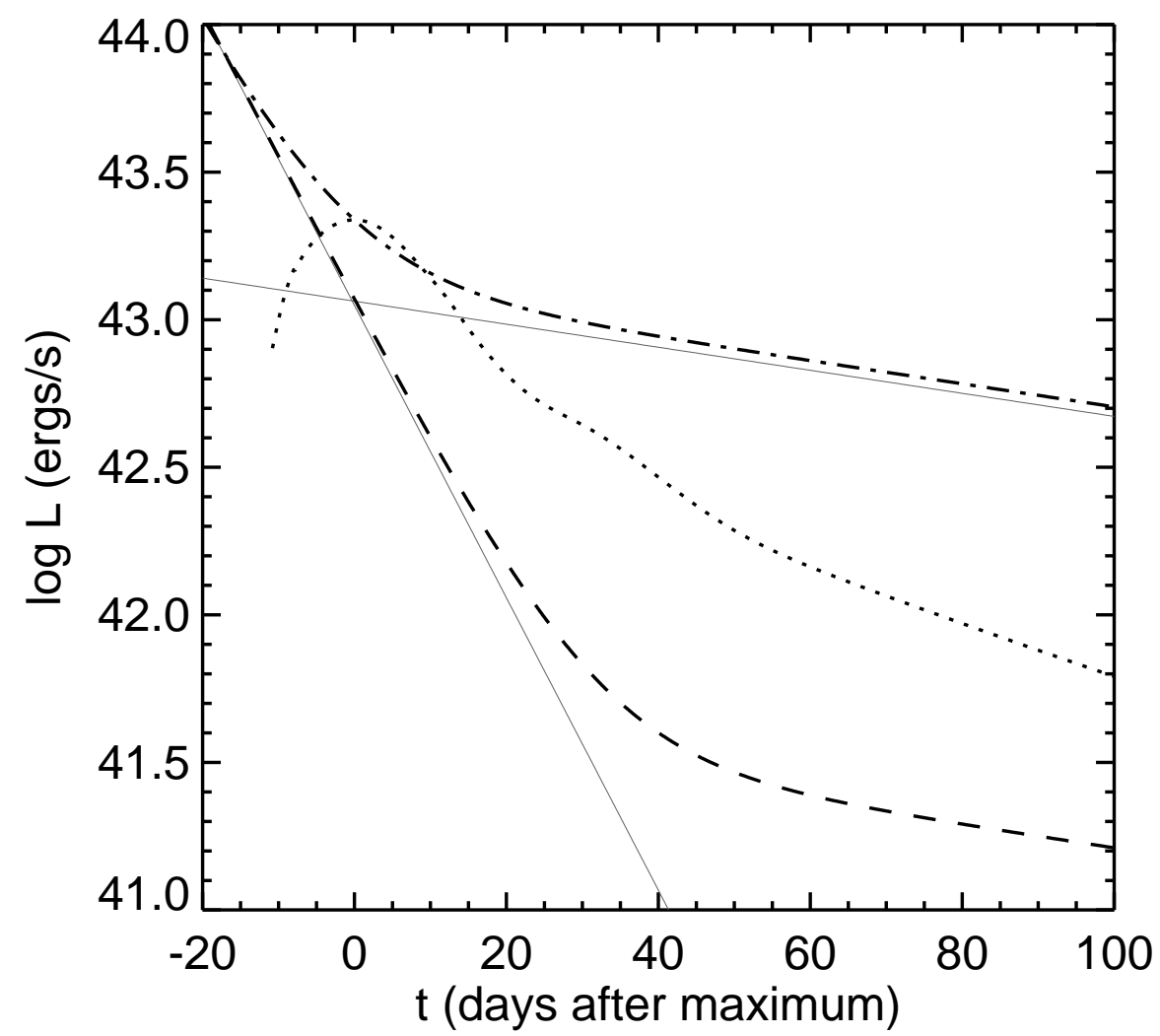

Fig. 2. Bolometric light curve of a SNIa. The dotted line is the observed bolometric light curve. The thin lines represent the ${ }^{56} \mathrm{Ni}$ and ${ }^{56} \mathrm{Co}$ decay lines, while the dashed line would be the expected curve if all $\gamma$-rays from the radioactive decay escape the ejecta and only the positrons are converted into optical emission. The dash-dotted line indicates the expectation of full trapping of all decay energy [23].

Correlations The SNIa light curve shape has been recognized as correlating with the peak luminosity [87]. This has become the linchpin for distance determinations using SNIa (see, e.g., the chapter by Perlmutter and Schmidt in this volume). The normalization of the peak luminosity allows the determination of cosmological parameters. However, the correlation is not as clear-cut as one would wish. There are three implementations $[83,88,91]$, which are currently not consistent with each other $[28,58]$.

There are other parameters which correlate with the peak luminosity of SNIa. They are the rise time to maximum [93], color near maximum light [88], line strengths of the primary $\mathrm{Ca}$ and $\mathrm{Si}$ absorption lines [79], the velocities as measured in Fe lines at late phases [70], the host galaxy morphology [101], and host 
galaxy colors $[16,43]$. There may be indications that the secondary peak in the I light curves and the shoulder in the bolometric light curves correlate with the absolute luminosity $[24,44,91]$.

With SN2000cx we now also have a clear example of an object which does not follow these simple rules. For most objects the rise and decline rates correlate fairly well [24,93], but SN2000cx violates this rule [66]. While it had a rapid rise, its light curve did decline more slowly than many other SNIa. It is, at the moment, not clear what fraction of SNIa show a similar behavior as the rise phase is often not observed and the correlations have been based on the decline rate. Nevertheless, the fact that for some SNIa the rise and decline rates do correlate $[24,93]$ hints at intrinsic differences within this class.

The physical reason for these correlations are not yet clear. Possibilities are differences in the amount ${ }^{56} \mathrm{Ni}$ synthesized and the distribution of the radioactive material, and hence the heating of the ejecta $[49,71,86]$.

\section{Bolometric Light Curves}

The connection of the observations to the explosion and radiation physics has to come through bolometric light curves as they represent the total energy output of a supernova. This integrated quantity is easily constructed from the available multi-filter photometry and is also rather easily extracted from model calculations. Detailed calculations of the emerging spectrum have proven difficult for all supernova types. While the physics of the radiation escape for core-collapse supernovae during the plateau phase, with their large envelopes and a rather well-defined photosphere (see, e.g., the chapter by Branch et al. in this volume), is fairly simple to calculate, possible chemical abundance mixing and deviations from spherical geometry can spoil direct comparison with the observations. For thermonuclear supernovae the spectrum formation is due to multiple scattering of the $\gamma$-rays in a non-thermal environment and hence the photons rather "leak" out at the surface than originate from a thermalized photosphere. Hence, the calculation of a filter light curve requires a detailed spectrum formation calculation which, in the case of SNIa, is currently impossible at the needed detail.

The temporal evolution of the integrated light gives a rather straight forward comparison between theory and observation. Important explosion parameters can be calculated from these integrated quantities. Foremost, the amount of energy available from the nucleosynthesis can be determined from bolometric light curves. The nickel masses derived for all supernovae are an essential input of the explosion models. So far, this observational input has been missing. It is foreseeable that in the near future this will change and observational constraints on the models will become available.

The bolometric light curve of SN1987A has been instrumental in decoding the various phases of the energy release (see Fig. 1 and $[72,111]$ ). The luminosity of the plateau phase indicates the size of the progenitor and the explosion energy, while the length of the plateau phase is mostly dominated by the mass of the exploded star [89]. For the core-collapse supernovae the luminosity on the 
radioactive tail, after the light curve leaves the plateau due to the recombination, together with the time since explosion gives the ${ }^{56} \mathrm{Ni}$ mass directly. This method has been employed for several supernovae and it could be shown that there are rather large differences among these objects. This is particularly interesting as it might provide insight into fall-back onto the forming neutron star.

It should be possible to combine some of these measurements into a picture of the explosion. One can investigate how the progenitor mass correlates with the explosion energy and the amount of fall-back onto the forming compact remnant. In extreme cases, it might be possible to show that a black hole formed in the explosion [6]. It is essential to show that the event has all the signatures of a massive stellar explosion to avoid confusion between different explosion types. Comparing the explosion parameters with direct information of the progenitor star, which are becoming available more often now (see, e.g., [102]), will be an important link between the post-event deductions and the models.

The bolometric light curves of thermonuclear supernovae can be used to extract physical parameters of the explosions (see, e.g., [118]). According to Arnett's rule $[2,85]$, the peak luminosity of SNIa reflects the amount of radioactive ${ }^{56} \mathrm{Ni}$ produced in the explosion. This fact has been used by Contardo et al. [24] (see also [23]) to derive the ${ }^{56} \mathrm{Ni}$ masses for several supernovae. They claim that there is a significant range of ${ }^{56} \mathrm{Ni}$ produced in the explosions (up to a factor of ten when extreme objects are included). A similar result was derived by Cappellaro et al. [17] based on the late light curves and the assumption that the $\mathrm{V}$ light curve can serve as a surrogate for the bolometric luminosity. Independent confirmation of this result comes from the observations of infrared spectra of SNIa [13].

The late decline can be further used to determine the escape fraction of the $\gamma$-rays. A steeper decline indicates a faster decrease of the column density. Three possible explanations for this effect could be: 1) different explosion energies, 2) a varying distribution of nickel in the explosion, or 3) differences in ejecta mass. All possibilities indicate fundamental variations in the explosions. Interestingly, the expansion velocity of the iron in the ejecta correlates with peak luminosity of SNIa [70], however not in the way expected from simple models. The brighter supernovae have larger expansion velocities indicating, for a fixed ejecta mass, a higher explosion energy. These are also the objects which produce more ${ }^{56} \mathrm{Ni}$ in the explosions. In this case, the distribution of the iron-peak elements must be different to explain the slower decline at late times [23]. An alternative explanation could be that the ejecta mass for SNIa is not the same in all events, quite a radical suggestion in view of the currently favored models of Chandrasekharmass white dwarf progenitors (see, e.g., [67]). Bolometric light curves of more objects will be needed to confirm such a result.

\section{Summary}

SN light curves are essential for our understanding of supernova physics. The acquisition of light curves has become relatively easy and the use of robotic 
telescopes and pipeline reductions will further advance. The success of recent supernova searches is increasing the available data set dramatically and we will soon be able to investigate detailed statistics on light curve parameters.

The variety of light curve shapes of core-collapse supernovae provides evidence for the many physical effects which can influence the outcome of these events. The rapid, and sometimes violent, evolution of their massive progenitor stars and their death within their cradle provides for vastly different environments and displays. We can still learn a great deal from detailed observations. The physics background has developed considerably over the past decade and the nearby example of SN1987A, offering extremely detailed observations, has led to major new insights into this phenomenon.

The short lifetime of core-collapse supernovae can be used to directly measure the star formation rates as a function of look back time (see, e.g., [25] and the chapter by Cappellaro in this volume). However, their light and color curves are required for a secure identification.

The more uniform appearance of thermonuclear type Ia explosions has been challenged by the observations in redder filter bands and additional examples. Although these objects still are rather uniform, their light curves have allowed us to show that significant variations in these explosions must exist. The correlation of the light curve shape with the luminosity has provided a convenient way to normalize these objects and make them the best cosmological distance indicator available at the moment (see the chapter by Perlmutter and Schmidt in this volume). Light curvesare at the heart of the cosmological applications of SNIa. It remains a major task to establish the physical understanding of these events in the coming years.

\section{References}

1. G. Aldering, R. Knop, P. Nugent: Astron. J. 119, 2110 (2000)

2. W.D. Arnett: Astrophys. J. 253, 785 (1982)

3. W.D. Arnett: Supernovae and Nucleosynthesis (Princeton University Press, Princeton 1996)

4. W.D. Arnett, J.N. Bahcall, R.P. Kirshner, S.E. Woosley: Ann. Rev. Astron. Astrophys. 27, 629 (1989)

5. T.S. Axelrod: In: Type I Supernovae, ed. by J.C. Wheeler (University of Texas, Austin 1980) p. 80

6. S. Balberg, L. Zampieri, S.L. Shapiro: Astrophys. J. 541, 860 (2000)

7. R. Barbon, F. Ciatti, L. Rosino: Astron. Astrophys. 116, 35 (1982)

8. R. Barbon, S. Benetti, E. Cappellaro, F. Patat, M. Turatto, T. Iijima: Astron. Astrophys. Suppl. Ser. 110, 513 (1995)

9. S. Benetti, M. Turatto, E. Cappellaro, I.J. Danziger, P.A. Mazzali: Mon. Not. R. Astron. Soc. 305, 811 (1999)

10. S. Benetti et al.: Mon. Not. R. Astron. Soc. 322, 361 (2001)

11. P.J. Benson et al.: Astron. J. 107, 1453 (1994)

12. P. Bouchet, M.M. Phillips, N.B. Suntzeff, C. Gouiffes. R. Hanuschik, D.H. Wooden: Astron. Astrophys. 245, 490 (1991) 
13. E.J.V. Bowers, W.P.S. Meikle, T.R. Geballe, N.A. Walton, P.A. Pinto, V.S. Dhillon, S.B. Howell, M.K. Harrop-Allin: Mon. Not. R. Astron. Soc. 290, 663 (1997)

14. D. Branch: Astrophys. J. 392, 35 (1992)

15. D. Branch, S.W. Falk, M.L. McCall, P. Rybski, A. Uomoto, B.J. Wills: Astrophys. J. 244, 780 (1981)

16. D. Branch, W. Romanishin, E. Baron: Astrophys. J. Lett. 465, L73 (1996)

17. E. Cappellaro, P.A. Mazzali, S. Benetti, I.J. Danziger, M. Turatto, M. Della Valle, F. Patat: Astron. Astrophys. 328, 203 (1998)

18. E. Cappellaro et al.: Astrophys. J. Lett. 549, L215 (2001)

19. N.N. Chugai: Sov. Astron. Lett. 16, 457 (1991)

20. A. Clocchiatti, J.C. Wheeler, E.S. Barker, A.V. Filippenko, T. Matheson, J.W. Liebert: Astrophys. J. 446, 167 (1995)

21. A. Clocchiatti et al.: Astrophys. J. 553, 886 (2001)

22. S.A. Colgate, A.G. Petschek, J.T. Kriese: Astrophys. J. Lett. 237, L81 (1980)

23. G. Contardo: Bolometric light curves of Type Ia Supernovae. PhD Thesis, Technical University, Munich (2001)

24. G. Contardo, B. Leibundgut, W.D. Vacca: Astron. Astrophys. 359, 876 (2000)

25. T. Dahlén, C. Fransson: Astron. Astrophys. 350, 349 (1999)

26. R. Diehl, F.X. Timmes: Pub. Astron. Soc. Pacific 110, 637 (1998)

27. V.T. Doroshenko, Yu.S. Efimov, N.M. Shakhovskoi: Astron. Lett. 21, 580 (1995)

28. P.S. Drell, T.J. Loredo, I. Wasserman: Astrophys. J. 530, 593 (2000)

29. R.G. Eastman, B.P. Schmidt, R.P. Kirshner: Astrophys. J. 466,, 911 (1996)

30. J.H. Elias, J.A. Frogel, J.A. Hackwell, S.E. Persson: Astrophys. J. Lett. 251, L13 (1981)

31. S.W. Falk, W.D. Arnett: Astrophys. J. Suppl. 33, 515 (1977)

32. A. Fassia et al.: Mon. Not. R. Astron. Soc. 318, 1093 (2000)

33. R.A. Fesen: Astrophys. J. Lett. 413, L109 (1993)

34. R.A. Fesen, R.H. Becker: Astrophys. J. 351, 437 (1990)

35. R.A. Fesen, D.M. Matonick: Astrophys. J. 407, 110 (1993)

36. R.A. Fesen et al.: Astron. J. 117, 725 (1999)

37. A.V. Filippenko: Ann. Rev. Astron. Astrophys. 35, 309 (1997)

38. A.V. Filippenko et al.: Astron. J. 104, 1534 (1992)

39. C. Fransson, A. Cassatella, R. Gilmozzi, R.P. Kirshner, N. Panagia, G. Sonneborn, W. Wamsteker: Astrophys. J. 336, 429 (1989)

40. C. Fransson, C. Kozma: Astrophys. J. Lett. 408, L25 (1993)

41. C. Fransson et al.: Astrophys. J. 572, 350 (2002)

42. G. Goldhaber et al.: Astrophys. J. 558, 359 (2001)

43. M. Hamuy, M.M. Phillips, J. Maza, N.B. Suntzeff, R.A. Schommer, R. Avilés: Astron. J. 109, 1 (1995)

44. M. Hamuy et al.: Astron. J. 112, 2408 (1996)

45. M. Hamuy et al.: Astrophys. J. 558, 615 (2001)

46. M. Hernandez et al.: Mon. Not. R. Astron. Soc. 319, 223 (2000)

47. W. Hillebrandt, J.C. Niemeyer: Ann. Rev. Astron. Astrophys. 38, 191 (2000)

48. P. Höflich: Astrophys. J. 443, 89 (1995)

49. P. Höflich, A.M. Khokhlov, J.C. Wheeler, M.M. Phillips, N.B. Suntzeff, M. Hamuy: Astrophys. J. Lett. 472, L81 (1996)

50. S. Jha et al.: Astrophys. J. Suppl. 125, 73 (1999)

51. R.P. Kirshner: In: Supernovae, ed. by A.G. Petschek (Springer, New York 1990) p. 59 
52. R.P. Kirshner et al.: Astrophys. J. 415, 589 (1993)

53. R.I. Klein, R.A. Chevalier: Astrophys. J. Lett. 223, L109 (1978)

54. K. Krisciunas et al.: Astron. J. 122, 1616 (2001)

55. B. Leibundgut: In: Circumstellar Media in Late Stages of Stellar Evolution, ed. by R. Clegg, I. Stevens, P. Meikle (Cambridge University Press, Cambridge 1994), p. 100

56. B. Leibundgut: In: The Lives of Neutron Stars, ed. by A. Alpar, Ü. Kiziloglu, J. van Paradijs (Kluwer, Dordrecht 1995) p. 3

57. B. Leibundgut: In: IAU Colloquium 145: Supernovae and Supernova Remnants, ed. by R. McCray, (Cambridge University Press, Cambridge 1996) p. 11

58. B. Leibundgut: Astron. Astrophys. Rev. 10, 179 (2000)

59. B. Leibundgut, P.A. Pinto: Astrophys. J. 401, 49 (1992)

60. B. Leibundgut, G.A. Tammann, R. Cadonau, D. Cerrito: Astron. Astrophys. Suppl. Ser. 89, 537 (1991a)

61. B. Leibundgut, R.P. Kirshner, P.A. Pinto, M.P. Rupen, R.C. Smith, J.E. Gunn, D.P. Schneider: Astrophys. J. 372, 531 (1991b)

62. B. Leibundgut et al.: Astron. J. 105, 301 (1993)

63. D.C. Leonard et al.: Pub. Astron. Soc. Pacific 114, 35 (2002)

64. J.R. Lewis et al.: Mon. Not. R. Astron. Soc. 266, L27 (1994)

65. W.D. Li, A.V. Filippenko, R.R. Treffers, A.G. Riess, J. Hu, Y. Qiu: Astrophys. J. 546, 734 (2001a)

66. W.D. Li et al.: Pub. Astron. Soc. Pacific 113, 1178 (2001b)

67. M. Livio: In: Type Ia Supernovae: Theory and Cosmology, ed. by J.C. Niemeyer, J.W. Truran, (Cambridge University Press, Cambridge 1999) p. 33

68. K.S. Long, W.P. Blair, W. Krzeminski: Astrophys. J. Lett. 340, L25 (1989)

69. K.S. Long, P.F. Winkler, W.P. Blair: Astrophys. J. 395, 632 (1992)

70. P.A. Mazzali, E. Cappellaro, I.J. Danziger, M. Turatto, S. Benetti: Astrophys. J. Lett. 499, L49 (1998)

71. P.A. Mazzali, K. Nomoto, E. Cappellaro, T. Nakamura, H. Umeda, K. Iwamoto: Astrophys. J. 547, 988 (2001)

72. R. McCray: Ann. Rev. Astron. Astrophys. 31, 175 (1993)

73. W.P.S. Meikle: Mon. Not. R. Astron. Soc. 314, 782 (2000)

74. N.V. Melova, D.Yu. Tsvetkov, S.Yu. Shugarov, V.F. Esipov, N.N. Pavlyuk: Astron. Lett. 21, 670 (1995)

75. P.A. Milne, L.-S. The, M. Leising: Astrophys. J. Suppl. 124, 503 (1999)

76. P.A. Milne, L.-S. The, M. Leising: Astrophys. J. 559, 1019 (2001)

77. M. Modjaz et al.Pub. Astron. Soc. Pacific 113, 308 (2001)

78. D.K. Nadyozhin: In: Supernovae and Cosmology, ed. by L. Labhardt, B. Binggeli, R. Buser (University of Basel, Basel 1998) p. 125

79. P. Nugent, M.M. Phillips, E. Baron, D. Branch, P. Hauschildt: Astrophys. J. Lett. 455, L147 (1995)

80. F. Patat, R. Barbon, R. Cappellaro, M. Turatto: Astron. Astrophys. Suppl. Ser. 98, 443 (1993)

81. F. Patat, R. Barbon, R. Cappellaro, M. Turatto: Astron. Astrophys. 282, 731 (1994)

82. F. Patat, R. Barbon, E. Cappellaro, M. Turatto: 1997, Astron. Astrophys. 317, $423(1997)$

83. S. Perlmutter et al.: Astrophys. J. 483, 565 (1997)

84. M.J. Pierce, G.H. Jacoby: Astron. J. 110, 2885 (1995)

85. P.A. Pinto, R.G. Eastman: Astrophys. J. 530, 757 (2000) 
86. P.A. Pinto, R.G. Eastman: New Astron. 6, 307 (2001)

87. M.M. Phillips: Astrophys. J. Lett. 413, L105 (1993)

88. M.M. Phillips, P. Lira, N.B. Suntzeff, R.A. Schommer, M. Hamuy, J. Maza: Astron. J. 118, 1766 (1999)

89. D.V. Popov: Astrophys. J. 414, 712 (1993)

90. M.W. Richmond, R.R. Treffers, A.W. Filippenko, Y. Paik: Astron. J. 112, $732(1996)$

91. A.G. Riess et al.: Astron. J. 116, 1009 (1998a)

92. A.G. Riess et al.: Astron. J. 117, 707 (1999a)

93. A.G. Riess et al.: Astron. J. 118, 2675 (1999b)

94. P. Ruiz-Lapuente, H.C. Spruit: Astrophys. J. 500, 360 (1998)

95. M.P. Rupen, J.H. van Gorkom, G.R. Knapp, J.E, Gunn, D.P. Schneider: Astron. J. 94, 61 (1987)

96. S. Ryder, L. Staveley-Smith, M.A. Dopita, R. Petre, E. Colbert, D. Malin, E. Schlegel: Astrophys. J. 416, 167 (1993)

97. M.E. Salvo, E. Cappellaro, P.A. Mazzali, S. Benetti, I.J. Danziger, F. Patat, M. Turatto: Mon. Not. R. Astron. Soc. 321, 254 (2001)

98. B.P. Schmidt et al.: Astron. J. 105, 2236 (1993)

99. B.P. Schmidt et al.: Astron. J. 107, 1444 (1994a)

100. B.P. Schmidt, R.P. Kirshner, B. Leibundgut, L.A. Wells, A.C. Porter, P. RuizLapuente, P. Challis, A.V. Filippenko: Astrophys. J. Lett. 434, L19 (1994b)

101. B.P. Schmidt et al.: Astrophys. J. 507, 46 (1998)

102. S.J. Smartt, G.F. Gilmore, N. Trentham, C.A. Tout, C.M. Frayn: Astrophys. J. Lett. 556, L29 (2001)

103. J. Sollerman, R.J. Cumming, P. Lundqvist: Astrophys. J. 493, 933 (1998a)

104. J. Sollerman, B. Leibundgut, J. Spyromilio: Astron. Astrophys. 337, 207 (1998b)

105. J. Sollerman, C. Kozma, C. Fransson, B. Leibundgut, P. Lundqvist, F. Ryde, P. Woudt: Astrophys. J. Lett. 537, L127 (2000)

106. W.B. Sparks, F.D. Maccetto, N. Panagia, F.R. Boffi, D. Branch, M.L. Hazen, M. Della Valle: Astrophys. J. 523, 585 (1999)

107. N.B. Suntzeff: In: IAU Colloquium 145: Supernovae and Supernova Remnants, ed. by R. McCray (Cambridge University Press, Cambridge 1996) p. 41

108. N.B. Suntzeff, P. Bouchet: Astron. J. 99, 650 (1990)

109. N.B. Suntzeff, S. Heathcote, W.G. Weller, N. Caldwell, J.P. Huchra: Nature 334, 135 (1988)

110. N.B. Suntzeff, M.M. Phillips, D.L. DePoy, J.H. Elias, A.R. Walker: Astron. J. 102, 1118 (1991)

111. N.B. Suntzeff, M.M. Phillips, D.L. DePoy, J.H. Elias, A.R. Walker: Astrophys. J. Lett. 384, L33 (1992)

112. N.B. Suntzeff et al.: Astron. J. 117, 1175 (1999)

113. M. Turatto, E. Cappellaro, R. Barbon, M. Della Valle, S. Ortolani, L. Rosino: Astron. J. 100, 771 (1990)

114. M. Turatto, E. Cappellaro, I.J. Danziger, S. Benetti, C. Gouiffes, M. Della Valle: Mon. Not. R. Astron. Soc. 262, 128 (1993)

115. M. Turatto, S. Benetti, E. Cappellaro, I.J. Danziger, M. Della Valle, C. Gouiffes, P.A. Mazzali, F. Patat: Mon. Not. R. Astron. Soc. 283, 1 (1996)

116. M. Turatto et al.: Astrophys. J. Lett. 498, L129 (1998)

117. M. Turatto, A. Piemonte, S. Benetti, E. Cappellaro, P.A. Mazzali, I.J. Danziger, F. Patat: Astron. J. 116, 2431 (1998)

118. W.D. Vacca, B. Leibundgut: Astrophys. J. Lett. 471, L37 (1996) 
119. S.D. Van Dyk et al.: Pub. Astron. Soc. Pacific 111, 313 (1999)

120. T. Wada, M. Ueno: Astron. J. 113, 231 (1997)

121. J.C. Wheeler, S. Benetti: In: Allen's Astrophysical Quantities, 4th edition, ed. by A.N. Cox (AIP Press, New York 2000) p. 451

122. S.W. Woosley, F.X. Timmes: 1996, Nucl. Phys. A 606, 137 (1996)

123. S.E. Woosley, T.A. Weaver: Ann. Rev. Astron. Astrophys. 24, 205 (1986)

124. J. Xu, A.P.S. Crotts, W.E. Kunkel: Astrophys. J. 451, 806 (1995) 